Provided for non-commercial research and education use. Not for reproduction, distribution or commercial use.

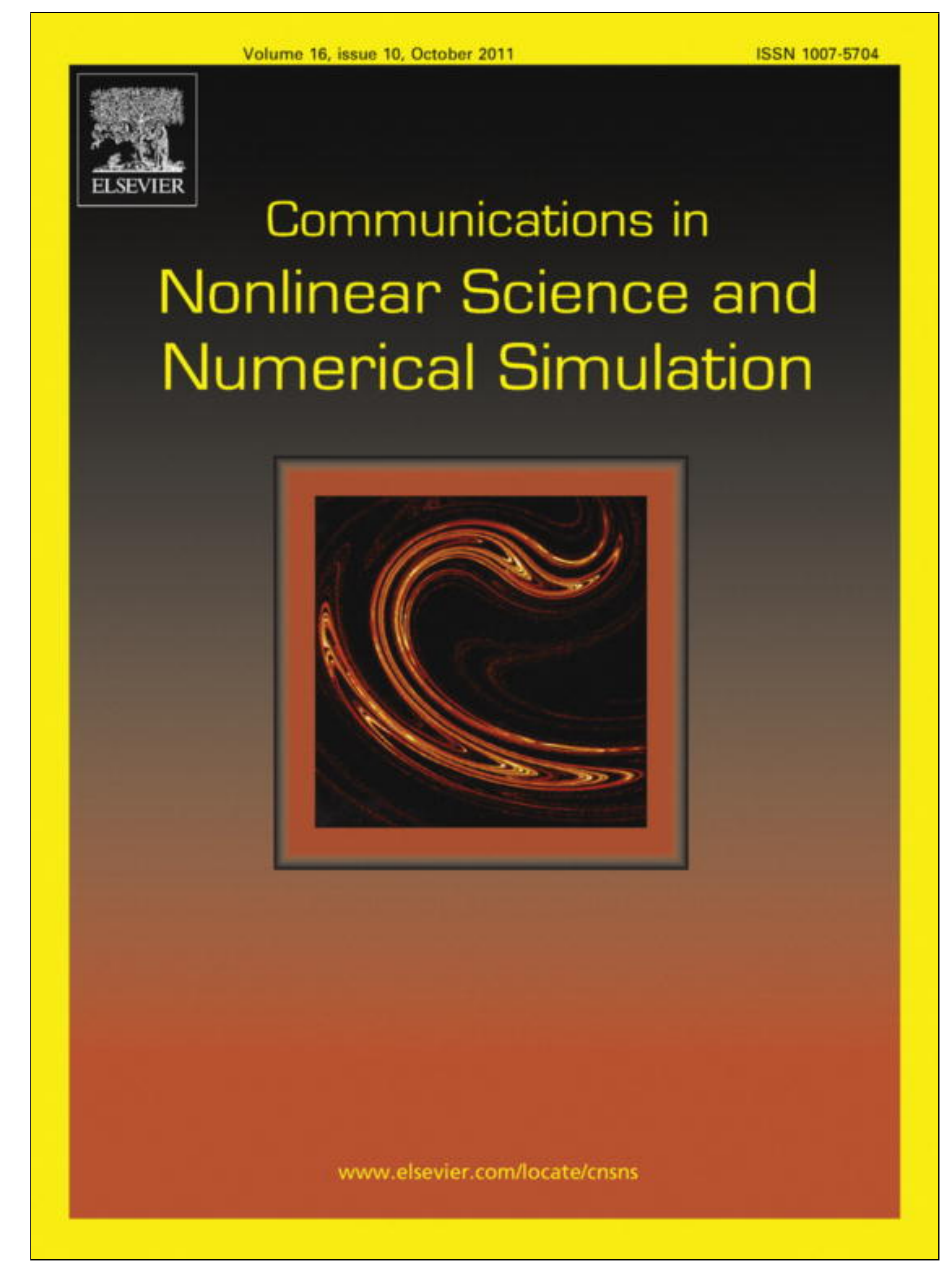

This article appeared in a journal published by Elsevier. The attached copy is furnished to the author for internal non-commercial research and education use, including for instruction at the authors institution and sharing with colleagues.

Other uses, including reproduction and distribution, or selling or licensing copies, or posting to personal, institutional or third party websites are prohibited.

In most cases authors are permitted to post their version of the article (e.g. in Word or Tex form) to their personal website or institutional repository. Authors requiring further information regarding Elsevier's archiving and manuscript policies are encouraged to visit:

http://www.elsevier.com/copyright 


\title{
Influence of diffusion of magnetic particles on stability of a static magnetic fluid seal under the action of external pressure drop
}

\author{
V. Polevikov ${ }^{a}$, L. Tobiska ${ }^{\mathrm{b}, *}$ \\ ${ }^{a}$ Department of Computational Mathematics, Belarusian State University, Independence Ave. 4, 220030 Minsk, Belarus \\ ${ }^{\mathrm{b}}$ Institut für Analysis und Numerik, Otto-von-Guericke-Universität Magdeburg, Postfach 4120, D-39016 Magdeburg, Germany
}

\section{A R T I C L E I N F O}

\section{Article history:}

Received 1 July 2010

Received in revised form 17 January 2011

Accepted 18 February 2011

Available online 27 February 2011

\section{Keywords:}

Magnetic fluid seal

Numerical simulation

Particle diffusion

Free surface

\begin{abstract}
A B S T R A C T
We derive a mathematical model for studying the stability of magnetic fluid seal under the action of external pressure drop in the static case. We propose a numerical algorithm for computing the free surface shape under the influence of diffusion of magnetic particles. We include also the case in which the particle concentration achieves its maximum corresponding to the dense packing of the particles. Numerical experiments for various sets of parameters give interesting insights in the dependence of the burst pressure on different parameters.
\end{abstract}

(c) 2011 Elsevier B.V. All rights reserved.

\section{Introduction}

Magnetic fluid seals are the best known and widely used technical application of magnetic fluids. Therefore, the development of an adequate mathematical model describing the relevant features of a magnetic fluid seal and numerical algorithms for its solution are of great importance, e.g. for the computer optimized design of magnetic fluid seals. The main objective of the paper is the development of such a realistic model. The burst external pressure drop and the critical velocity of rotation of the shaft are the main characteristics of a magnetic fluid seal. They are defined essentially by the shape of the sealing layer which is under the action of capillary, magnetic, centrifugal and pressure forces.

An acceptable mathematical model of a magnetic fluid seal which takes into account the free surface deformation has been developed and realized in [1,2]. It is based on the assumption that the pole piece of an annular permanent magnet being concentrator of magnetic flux in the seal gap is of hyperbolic shape. This allows to find an exact solution of Maxwell equations for the magnetic field in the gap. Later this assumption has been used in [3-5]. The stability of a magnetic fluid seal has been investigated numerically in [1-4] under the assumption of a uniform concentration of ferromagnetic particles in the fluid. In [1,2] the stability of rotating shaft has been studied at zero pressure drop whereas in [3] static magnetic fluid seal under the action of a pressure drop has been considered. In [4], the stability problem of a magnetic fluid seal has been solved in a more general statement; the dynamic magnetic fluid seal has been considered under the action of both centrifugal forces and a pressure drop.

As the next step toward the development of a realistic mathematical model we consider in this paper the influence of the magnetic particle diffusion process on the stability of the seal. In [5] an attempt was made to investigate the effect of particle concentration on the stability of a magnetic fluid seal however a predefined free surface shape independent of external forces

\footnotetext{
* Corresponding author.

E-mail address: tobiska@mathematik.uni-magdeburg.de (L. Tobiska).
} 
has been assumed. We will consider the static magnetic fluid seal under an external pressure drop and extend the model and results obtained for a uniform particle concentration in [3] by taking particle diffusion into consideration.

\section{Mathematical model}

\subsection{Statement of the problem}

We will study the behaviour of a magnetic fluid seal as shown in Fig. 1 in the narrow profiled gap between two coaxial surfaces of revolution. The magnetic fluid between the shaft and the polar head forms a hermetic barrier isolating regions at different pressures. This pressure drop and centrifugal forces try to push out the magnetic fluid which is hold in the gap by the high-gradient magnetic field. In previous studies [1-4,6], the particle diffusion leading to a highly nonuniform concentration of particles has been neglected. In this paper, a mathematical model is developed taking the particle diffusion into consideration. In the static case, the model is used to investigate the stability of the magnetic fluid seal.

\subsection{General equations}

We consider the unknown fluid domain $\Omega_{f}$ of the ferrofluid inside of a larger domain $\Omega$. The Maxwell equation for nonconducting media are given by

$$
\operatorname{curl} \mathbf{H}=\mathbf{0}, \quad \operatorname{div} \mathbf{B}=0, \quad \mathbf{B}=\mu_{0}(\mathbf{H}+\mathbf{M}) \quad \text { in } \Omega,
$$

with the magnetic field strength $\mathbf{H}$, the magnetic induction $\mathbf{B}$, the magnetization $\mathbf{M}$, and $\mu_{0}=4 \pi \times 10^{-7} \mathrm{H} / \mathrm{m}$. In a region of nonmagnetizable material, e.g. air, we have $\mathbf{M}=\mathbf{0}$. We assume that the magnetization $\mathbf{M}$ of the ferrofluid is parallel to the magnetic field $\mathbf{H}$ and that it follows the nonlinear Langevin magnetization law for dilute fluids

$$
M(H, C(H))=\frac{M_{s}}{C_{0}} C(H) L(\zeta H), \quad L(t)=\operatorname{coth} t-\frac{1}{t},
$$

with the saturation magnetization $M_{s}$, the particle concentration $C$, the mean concentration $C_{0}$ corresponding to a uniform particle distribution, the Langevin parameter $\zeta=\mu_{0} m_{m} /(k T), m_{m}$ the magnetic moment of a particle, $k=1.3806568 \times 10^{-23} \mathrm{~J} /$ K the Boltzmann constant, $T$ the particle temperature, $H=|\mathbf{H}|$, and $M=|\mathbf{M}|$.

The hydrodynamical properties of the ferrofluid are described by the incompressible Navier-Stokes equations

$$
\begin{aligned}
& \rho\left(\mathbf{v}_{t}+(\mathbf{v} \cdot \nabla) \mathbf{v}\right)-\eta \Delta \mathbf{v}+\nabla p=\rho \mathbf{g}+\mu_{0} M \nabla H \quad \text { in } \Omega_{f}, \\
& \operatorname{div} \mathbf{v}=0 \text { in } \Omega_{f},
\end{aligned}
$$

where $\rho$ denotes the fluid density, $\mathbf{v}$ the velocity, $\eta$ the dynamic viscosity, $\mathbf{g}$ the acceleration of gravity, and $p=p_{f}+p_{m}$ the (composite) pressure with the thermodynamic pressure $p_{f}$ in the ferrofluid and the fluid magnetic pressure

$$
p_{m}=\mu_{0} \int_{0}^{H} M\left(H^{\prime}, C\left(H^{\prime}\right)\right) d H^{\prime}
$$

We neglect the flow outside of $\bar{\Omega}_{f}$ and assume constant pressure fields in the two different hermetically separated regions.

Under the assumption that the magnetic particles are of spherical form and of equal size, the mass conservation of magnetic particles can be written in the form [7,8]

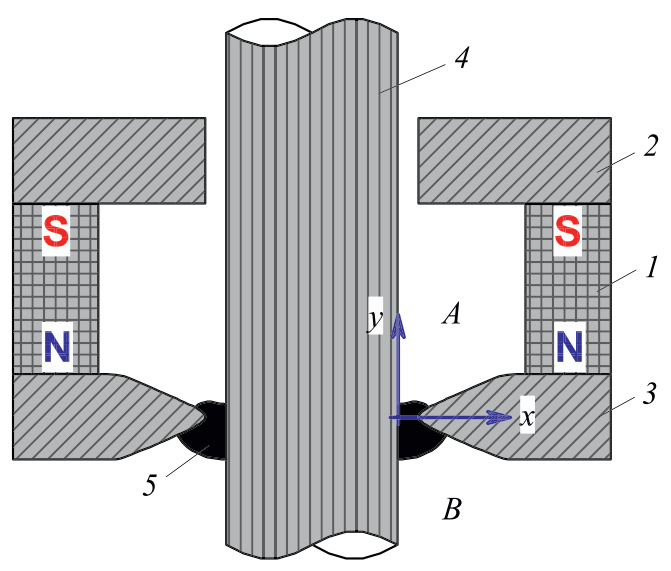

Fig. 1. Schematic representation of the magnetic fluid seal: 1 - magnet, 2 - core, 3 - magnetic flux concentrator, 4 - shaft, 5 - magnetic fluid, A - region of high pressure, $\mathrm{B}$ - region of low pressure. 


$$
\frac{\partial C}{\partial t}+\mathbf{v} \cdot \nabla C=D \nabla \cdot\left(\nabla C-C L(\zeta H) \nabla(\zeta H)-C \frac{m \mathbf{g}}{k T}\right) \text { in } \Omega_{f}
$$

where $D$ is the diffusion coefficient and $m$ the mass of a particle. The system (1)-(5) is additionally coupled by the force balance at the two free surfaces between the ferrofluid and the surrounding media. Let $\sigma$ denote the stress tensor given by

$$
\boldsymbol{\sigma}(\mathbf{v}, p, \mathbf{H})=2 \eta \mathbb{D}(\mathbf{v})-\left(p+\frac{\mu_{0}}{2} H^{2}\right) \llbracket+\mathbf{B} \otimes \mathbf{H},
$$

with the identity $\llbracket$ and velocity deformation tensor $\mathbb{D}(\mathbf{v})=\left(\nabla \mathbf{v}+\nabla \mathbf{v}^{T}\right) / 2$. Then, the force balance on the free surfaces $\Gamma_{f}^{i} \subset \partial \Omega_{f}, i=1,2$, becomes

$$
\left(\boldsymbol{\sigma}^{\text {out }}\left(\mathbf{0}, p_{i}, \mathbf{H}\right)-\boldsymbol{\sigma}^{\text {in }}(\mathbf{v}, p, \mathbf{H})\right) \mathbf{n}=\alpha \mathcal{K} \mathbf{n}, \quad \text { on } \Gamma_{f}^{i}, \quad i=1,2 .
$$

Here, $\mathbf{n}$ is the outward unit normal with respect to $\Omega_{f}, p_{i}$ the pressure of the $\Gamma_{f}^{i}$ surrounding region, $\alpha>0$ the surface tension coefficient, and $\mathcal{K}$ the sum of principal curvature. Multiplying (7) by the normal vector $\mathbf{n}$, using the continuity of the normal component of $\mathbf{B}$ and the tangential components of $\mathbf{H}$ over the free surfaces, we end up with the Young Laplace equation

$$
-2 \eta \mathbf{n} \cdot \mathbb{D}(\mathbf{v}) \mathbf{v}+p_{f}+p_{m}+\frac{\mu_{0}}{2}(\mathbf{M} \cdot \mathbf{n})^{2}=p_{i}+\alpha \mathcal{K} \quad \text { on } \Gamma_{f}^{i}, \quad i=1,2 .
$$

The coupled highly nonlinear system of partial differential equations have to be complemented by suitable boundary conditions and the condition that the fluid volume is preserved.

\section{Approximation}

\subsection{Model assumptions}

In this section we describe our assumptions leading to an approximated model for the stability of a magnetic fluid seal in the static case. Based on the assumption that the gap width is small compared to the radius of the rotary shaft we will use a plane $2 \mathrm{~d}$ approximation as already discussed in [4,9-11].

Neglecting the gravitational force, the dynamic Navier-Stokes equations ( 3 ) and (4) reduce to the hydrostatic limit $\mathbf{v}=\mathbf{0}$ and $p_{f}=0$. In this case the steady-state dimensionless concentration problem $(t \rightarrow \infty, \mathbf{v}=0)$ in the cross section $\Omega_{F}$ can be formulated in the form

$$
\begin{aligned}
& \nabla \cdot(\nabla C-C \nabla(\ln \varphi))=0 \quad \text { in } \Omega_{F}, \\
& \frac{\partial C}{\partial n}-\frac{\partial(\ln \varphi)}{\partial n} C=0 \quad \text { on } \Gamma=\partial \Omega_{F}, \\
& \frac{1}{\left|\Omega_{F}\right|} \int_{\Omega_{F}} C d \Omega_{F}=1,
\end{aligned}
$$

where

$$
\varphi(\zeta H)=\exp \int_{0}^{\zeta H} L(\gamma) d \gamma=\frac{\sinh (\zeta H)}{\zeta H} .
$$

$\left|\Omega_{F}\right|$ denotes the area of $\Omega_{F}$, and $C$ is normalized such that the uniform particle distribution corresponds to $C=C_{0}=1$. As shown in [8], there is an analytical solution of the problem (9)-(11) given by

$$
C(x, y)=\frac{\left|\Omega_{F}\right|}{\int_{\Omega_{F}} \varphi(\zeta H) d \Omega_{F}} \varphi(\zeta H(x, y)) .
$$

As a consequence, isolines of the magnetic field strength are also isolines of the particle concentration. In strong fields, it can happen that the particle concentration computed by formula (12) gives concentration values larger than its maximum value defined by the dense packing of particles. In this case a Stefan-type diffusion problem arises [8] and we subdivide the domain $\Omega_{F}$ into a domain $\Omega_{1}$ where $C<C_{\max }$ and a domain $\Omega_{2}=\Omega_{F} \backslash \Omega_{1}$ where $C=C_{\max }$. We solve the particle concentration equation on the 'unknown' subdomain $\Omega_{1} \subset \Omega_{F}$. The corresponding solution becomes

$$
C(x, y)=\left\{\begin{array}{cc}
K \varphi(\zeta H(x, y)) & \text { in } \Omega_{1}, \\
C_{\max } & \text { in } \Omega_{2} .
\end{array}\right.
$$

Since the curve separating the two domains is an isoline $C=C_{\max }$ of the particle concentration and an isoline of the field strength $H=H_{0}$, respectively, we have two requirements to determine the constant $K$ in (13) and the subdomains $\Omega_{1}, \Omega_{2}$ :

$$
\begin{aligned}
& \frac{1}{\left|\Omega_{F}\right|} \int_{\Omega_{F}} C d \Omega_{F}=K \int_{\Omega_{1}} \varphi(\zeta H) d \Omega_{1}+C_{\max }\left(\left|\Omega_{F}\right|-\left|\Omega_{1}\right|\right)=1, \\
& K \varphi\left(\zeta H_{0}\right)=C_{\max } .
\end{aligned}
$$

The solution of the problem (14) and (15) can be determined, for example, by the bisection method. 
In seal gaps, the magnetic field strength values reach $H \sim 10^{6} \mathrm{~A} / \mathrm{m}$ and the stress gradient values $|\nabla H| \sim 10^{9} \mathrm{~A} / \mathrm{m}^{2}$, see [12]. Therefore, we can neglect gravity effects on the free surface since the magnetic body force $\mu_{0} M \nabla H$ is two orders of magnitude greater than the gravity force $\rho \mathbf{g}$. The same reason allows us to neglect also capillary forces and magnetic jumps on the free surface. Then, the Young-Laplace Eq. (8) reduces to the algebraic equation

$$
\mu_{0} \int_{0}^{H_{i}} M\left(H^{\prime}, C\left(H^{\prime}\right)\right) d H^{\prime}+\text { const }=p_{i}, \quad i=1,2,
$$

where the integral can be determined analytically thanks to the analytic expression for the particle concentration along the free surface. We get

$$
\frac{\mu_{0} M_{s} K}{\zeta}\left(\varphi\left(\zeta H_{i}\right)-1\right)+\text { const }=p_{i}, \quad i=1,2 .
$$

Finally, we assume that the concentrator has a hyperbolic shape of dimensionless form

$$
y^{2}=\tan ^{2} \beta\left(x^{2}-1\right)
$$

as shown in Fig. 2.

When the magnetic field in the gap exceeds considerably the magnetization of the fluid (as it is the case in magnetic fluid seals), the local change of the magnetic field strength due to magnetization of the fluid can be neglected. Now, using elliptic cylinder coordinates we find an analytical expression for the magnetic field strength [1-4]:

$$
H(x, y)=\frac{H_{c} \sin \beta}{\sqrt[4]{\left(\left(x^{2}+y^{2}\right) \cos ^{2} \beta+1\right)^{2}-4 x^{2} \cos ^{2} \beta}},
$$

where $H_{c}$ denotes the magnetic intensity at the polar head.

\subsection{Computational algorithm}

We recall that our assumptions guarantee that the free surface shapes coincide with the isolines of the magnetic field strength. From (17) we get the explicit representations for the isolines $H(x, y)=H_{k}=H_{c} \sin \beta / \sigma_{k}$

$$
\begin{aligned}
& y=f\left(x, \sigma_{k}\right)= \pm \frac{1}{\cos \beta} \sqrt{\sqrt{\sigma_{k}^{4}+4 x^{2} \cos ^{2} \beta}-1-x^{2} \cos ^{2} \beta}, \\
& 0 \leqslant x \leqslant x_{k}=\sqrt{\sigma_{k}^{2}+\cos ^{2} \beta},
\end{aligned}
$$

i.e. the Cassinian ovals which are indicated in Fig. 3. Increasing the pressure drop the Bernoulli lemniscate describes the limit position of the free surface $\gamma_{1}$ before the seal collapses. This position corresponds to $\sigma_{1}=1$ and is independent of the fluid

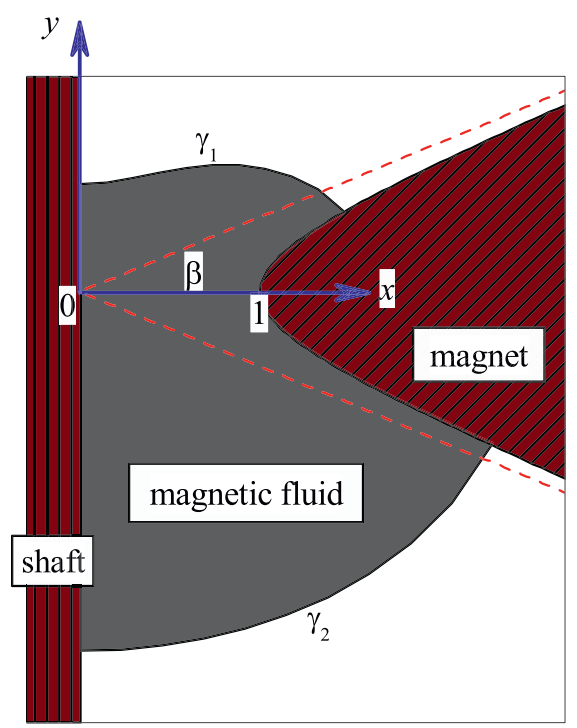

Fig. 2. Illustration to the problem statement: $\gamma_{1}$ - free surface on the high pressure region, $\gamma_{2}$ - free surface on the low pressure region, dashed lines asymptotes to the concentrator hyperbola, $\beta=$ half-angle between the asymptotes. 


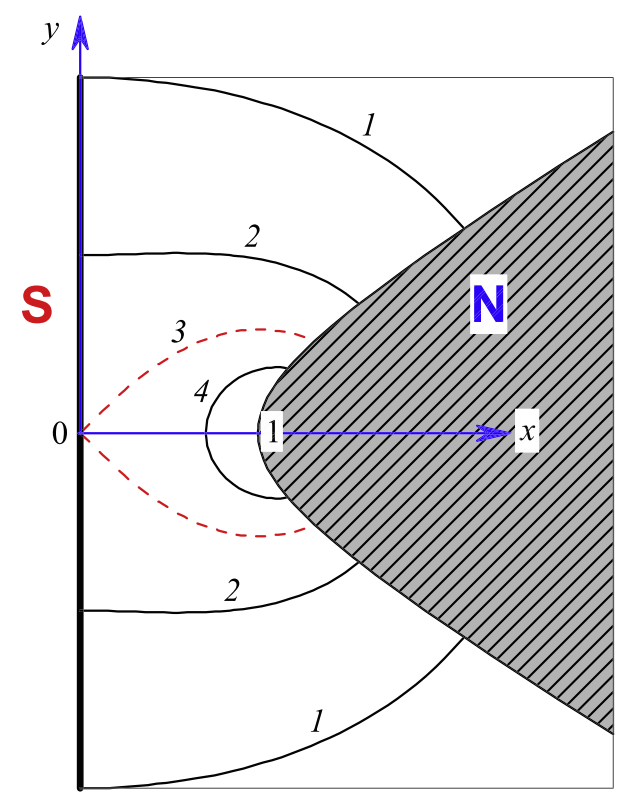

Fig. 3. Isolines of the magnetic field strength in the seal gap (Cassinian ovals): $1,2-H_{k}<H_{c} \sin \beta, 3-H_{k}=H_{c} \sin \beta$ (Bernoulli lemniscate), 4- $H_{k}>H_{c} \sin \beta$.

volume provided that the fluid volume is large enough to cover the area between $\pm \gamma_{1}$. The critical position of the free surface $\gamma_{2}$ is described by the equation

$$
\begin{aligned}
& y=f\left(x, \sigma_{2}\right)=-\frac{1}{\cos \beta} \sqrt{\sqrt{\sigma_{2}^{4}+4 x^{2} \cos ^{2} \beta}-1-x^{2} \cos ^{2} \beta}, \\
& 0 \leqslant x \leqslant x_{2}=\sqrt{\sigma_{2}^{2}+\cos ^{2} \beta}, \quad \sigma_{2}=\frac{H_{c} \sin \beta}{H_{2}}
\end{aligned}
$$

and is fixed by the given fluid volume $V$ which satisfies

$$
\begin{aligned}
V= & \int_{0}^{x_{1}} f\left(x, \sigma_{1}\right) d x-\frac{1}{2} \tan \beta\left\{x_{1} \sqrt{x_{1}^{2}-1}-\ln \left(x_{1}+\sqrt{x_{1}^{2}-1}\right)\right\} \\
& -\int_{0}^{x_{2}} f\left(x, \sigma_{2}\right) d x+\frac{1}{2} \tan \beta\left\{x_{2} \sqrt{x_{2}^{2}-1}-\ln \left(x_{2}+\sqrt{x_{2}^{2}-1}\right)\right\} .
\end{aligned}
$$
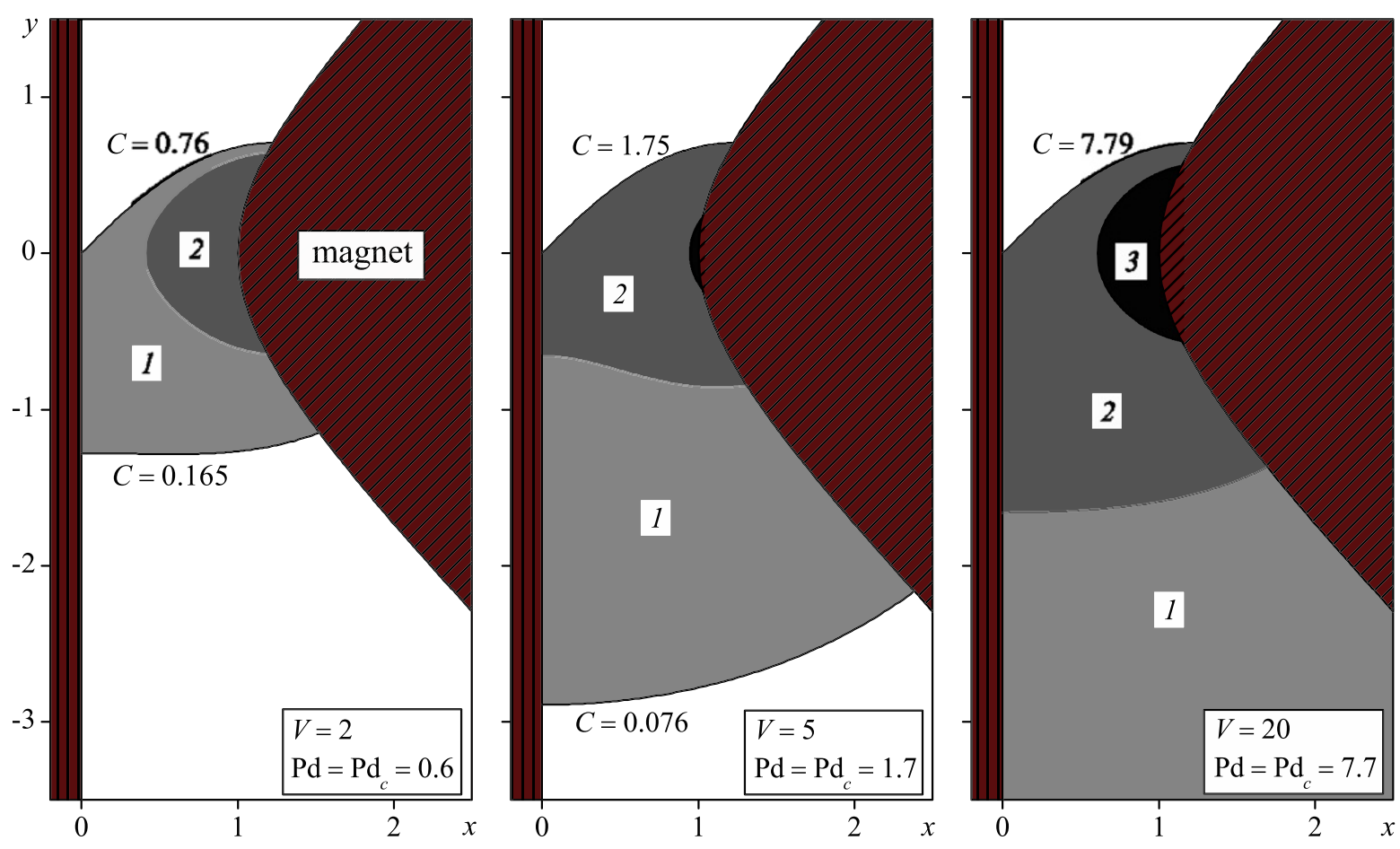

Fig. 4. Critical position of the magnetic fluid bridge and distribution of the particle concentration: $1-C<1,2-1<C<C_{\max }, 3-C=C_{\max }$. 


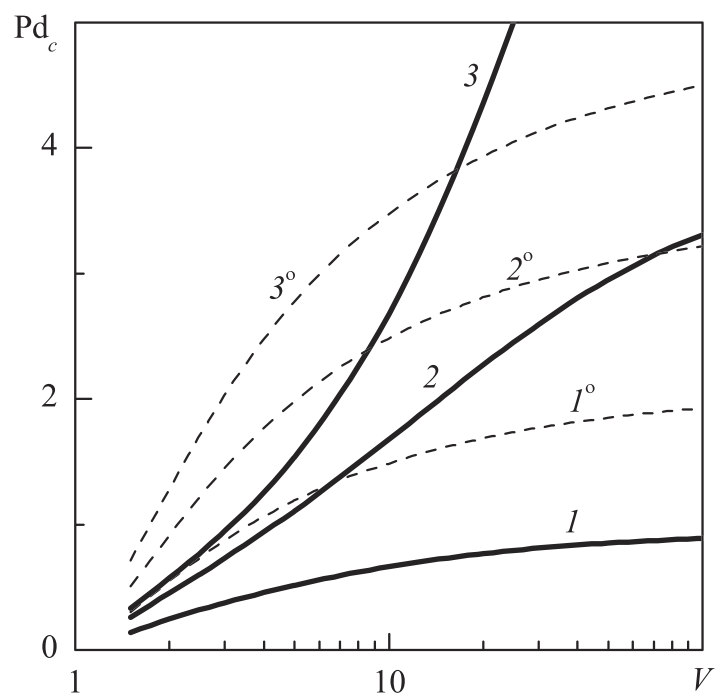

Fig. 5. Burst pressure drop depending on the fluid volume $V: 1-A=3,2-A=5,3-A=7$, in comparison to dependences $1^{\circ}, 2^{\circ}$, $3^{\circ}$ to the uniform particle concentration $C=1$.

The unknown constant $\sigma_{2}$ in (20), (21) can be determined from (22) by the bisection method. Then, the critical value for the dimensionless pressure drop $\mathrm{Pd}=m_{m}\left(p_{1}-p_{2}\right) /\left(k T M_{s}\right)$ follows from (16)

$$
\operatorname{Pd}_{c}=K\left\{\varphi(A \sin \beta)-\varphi\left(\frac{A \sin \beta}{\sigma_{2}}\right)\right\}, \quad A=\zeta H_{c} .
$$

\section{Numerical results}

First we show in Fig. 4 the numerical results for fixed values $A=10, \beta=45^{\circ}, C_{\max }=15$, and different fluid volume $V=2$, $V=5, V=20$. We see that with increasing fluid volume regions with dense packing appear. Looking to the particle concentration along $\gamma_{1}$ and $\gamma_{2}$, we see that with increasing volume the difference $c_{\gamma_{1}}-c_{\gamma_{2}}$ (which is proportional to Pd) increases too. This behaviour can also detected in Fig. 5 in which the critical pressure drop over the volume is presented for three different values of $A, A=3, A=5, A=7$, and the fixed values $\beta=45^{\circ}, C_{\max }=15$. This figure also demonstrates the differences caused by taking into account the diffusion process of particle by comparing the results with previous ones obtained in [3]. It is found experimentally in [13,14] that the redistribution of concentration due to the magnetophoretic transfer of particles results in a time-dependent sealing ability of magnetic-fluid shaft seal; the critical pressure drop increases appreciably as time grows. Fig. 5 shows that this is true provided that the volume $V$ is sufficiently large.

\section{Conclusions}

A mathematical model to study the stability of a magnetic fluid seal under the action of an external pressure drop has been developed and used for computing numerically the free surface shape. It has been demonstrated that there is a non-neglectable influence of the particle diffusion on the burst pressure, in particular, when for larger fluid volume the particle concentration achieves its maximum due to the dense packing of particles.

\section{References}

[1] Vislovich A, Polevikov V. Effect of the centrifugal and capillary forces on the free surface shape of the free surface of a magnetic liquid seal. Magnetohydrodynamics 1994;30:67-74.

[2] Vislovich A, Polevikov V. Concerning numerical simulation of the failure of a magnetic-fluid seal with a rotary outer profiled cylinder. J Eng Phys Thermophys 1997;70:107-12.

[3] Polevikov V. Stability of a static magnetic fluid seal under the action of an external pressure drop. Fluid Dynam 1997;32:457-61.

[4] Polevikov V, Tobiska L. Modeling of a dynamic magneto-fluid seal in the presence of a pressure drop. Fluid Dynam 2001;36:890-8.

[5] Krakov M, Nikiforov I. Influence of the shaft rotation on the stability of magnetic fluid shaft seal characteristics. Magnetohydrodynamics 2008;44:401-8.

[6] Budnik A, Polevikov V. Modeling the free surface of ring magneto-fluid packings with the help of the boundary-element method. Differ Equat 1992;28:940-4.

[7] Bashtovoi V, Polevikov V, Suprun A, Stroots A, Beresnev S. Influence of Brownian diffusion on the statics of magnetic fluid. Magnetohydrodynamics 2007;43:17-25.

[8] Polevikov V, Tobiska L. On the solution of the steady-state diffusion problem for ferromagnetic particles in a magnetic fluid. Math Model Anal 2008;13:233-40. 
[9] T. Mitkova, Lösbarkeit und FE-Approximation eines mathematischen Modells für die Strömung in Magnetfluiddichtungen, Ph.D. Thesis, Otto von Guericke University Magdeburg, 2004.

[10] Lavrova O, Matthies G, Mitkova T, Polevikov V, Tobiska L. Finite element methods for coupled problems in ferrohydrodynamics. In: Bänsch E, editor. Challenges in scientific computing. Springer-Verlag; 2002. p. 161-83.

[11] Lavrova O, Matthies G, Mitkova T, Polevikov V, Tobiska L. Numerical treatment of free surface problems in ferrohydrodynamics. J Phys Condens Matter 2006;18:S2657-69.

[12] Berkovsky B, Medvedev V, Krakov M. Magnetic fluids: engineering applications. Oxford: Oxford University Press; 1993.

[13] Taketomi S. Motion of ferrite particles under a high gradient magnetic field in a magnetic fluid shaft seal. J Appl Phys 1980;19:1929-36.

[14] Anton I, Vekash L, Potents I, Bika D. Behaviour of magnetic liquids in an inhomogenous magnetic field. Magnetohydrodynamics 1985;21:213-8. 